\title{
Blood Oxygen Level-Dependent Activation of the Primary Visual Cortex Predicts Size Adaptation Illusion
}

\author{
Arezoo Pooresmaeili, ${ }^{1}$ Roberto Arrighi, ${ }^{2,3}$ Laura Biagi, ${ }^{1}$ and Maria Concetta Morrone ${ }^{1,4}$ \\ ${ }^{1}$ Scientific Institute Stella Maris, 56018 Pisa, Italy, ${ }^{2}$ Institute of Neurosciences, National Research Council, 56124 Pisa, Italy, ${ }^{3}$ Department of Neuroscience, \\ Psychology, Pharmacology, and Child Health, University of Florence, 50121 Florence, Italy, and ${ }^{4}$ Department of Translational Research on New \\ Technologies in Medicine and Surgery, University of Pisa, 56126 Pisa, Italy
}

In natural scenes, objects rarely occur in isolation but appear within a spatiotemporal context. Here, we show that the perceived size of a stimulus is significantly affected by the context of the scene: brief previous presentation of larger or smaller adapting stimuli at the same region of space changes the perceived size of a test stimulus, with larger adapting stimuli causing the test to appear smaller than veridical and vice versa. In a human fMRI study, we measured the blood oxygen level-dependent activation (BOLD) responses of the primary visual cortex (V1) to the contours of large-diameter stimuli and found that activation closely matched the perceptual rather than the retinal stimulus size: the activated area of V1 increased or decreased, depending on the size of the preceding stimulus. A model based on local inhibitory V1 mechanisms simulated the inward or outward shifts of the stimulus contours and hence the perceptual effects. Our findings suggest that area V1 is actively involved in reshaping our perception to match the short-term statistics of the visual scene.

\section{Introduction}

Adaptation is a primary function of perceptual systems that tunes perceptual mechanisms to the statistical content of the retinal images (Shapley and Enroth-Cugell, 1984; Barlow, 1990; Blakemore et al., 1990; Sharpee et al., 2006; Clifford et al., 2007; Kohn, 2007), maximizing sensitivity to more probable events at the expense of rare ones. Although adaptation clearly improves coding efficiency, it often leads to an "illusory" perception that does not correspond to the physical stimulus (McCollough, 1965; Blakemore and Sutton, 1969; Gregory, 1997; Carandini, 2000; for review, see Thompson and Burr, 2009). Perception of size-a crucial task of our visual system - is not an exception to this rule because object size is rarely perceived veridically: spatial context (Leibowitz et al., 1969; Massaro and Anderson, 1971), vergence (Arnold et al., 2008), and apparent distance (Kaufman and Rock, 1962) all affect perceived size generating strong illusion.

Until recently, it has been considered that size perception is a high-level function, probably mediated by specific cortical areas selective to objects and object size (Ungerleider and Bell, 2011; for review, see Kourtzi and Connor, 2011; Konkle and Oliva, 2012). It has also been shown that the visual system has a double representation of size: one for conscious perception and one for the action system, which is less prone to illusion. The two size systems can be dissociated in the laboratory under particular conditions,

\footnotetext{
Received April 27, 2013; revised Aug. 2, 2013; accepted Aug. 21, 2013.

Author contributions:A.P., R.A., and M.C.M. designed research; A.P., R.A., and L.B. performed research; A.P., R.A., and M.C.M. wrote the paper.

This study has been supported by the Italian Ministry of University and Research and by European Community Projects "STANIB" and "ECSPLAIN" (FP7 ERC Advanced Grant).

The authors declare no competing financial interests.

Correspondence should be addressed to Concetta Morrone, Via Moruzzi 1, 56124 Pisa, Italy. E-mail: concetta@in.cnr.it.

DOI:10.1523/JNEUROSCI.1770-13.2013

Copyright $\odot 2013$ the authors $\quad 0270-6474 / 13 / 3315999-10 \$ 15.00 / 0$
}

reinforcing the idea of a high-level size representation for conscious size perception (Goodale et al., 1991; Aglioti et al., 1995).

Recently, the view that size constancy is only a high-level function has been challenged by two reports. One showed that the apparent size of an object activates different primary visual cortex (V1) representations, if displayed together with perspective visual cues (Murray et al., 2006; Fang et al., 2008). The other evidence uses the classic afterimage apparent size, which changes with vergence and fixation distance: V1 activity modulates with the perceived rather than the retinotopic stimulus size (Sperandio et al., 2012). Although both these experiments provide strong evidence for the involvement of V1 activity in size illusions, the BOLD effect in V1 in the experiment by Murray et al. (2006) could originate from pictorial cues driving distant neuronal process (Gilbert and Li, 2012) and in the study by Sperandio et al. (2012) could originate by eye-positional signals because these are known to strongly modulate V1 (Trotter and Celebrini, 1999; Andersson et al., 2007).

The effect of nonsimultaneous context, or adaptation, on size perception is less well documented than spatial context, although adaptation aftereffects are usually strong and not related to neuronal fatigue (Thompson and Burr, 2009). Importantly, adaptation does not rely on spatial contextual cues, such as depth or vergence, that originate in higher visual areas and is therefore optimally suited to test the role of early visual areas in the perception of object size. Here, we used an adaptation technique to high-pass images of circles that have the advantage that size cannot be deduced by energy at low spatial frequencies. These classes of stimuli, that induce the well known Craik-O'Brien-Cornsweet illusion, elicit localized activity in V1 in correspondence to the edges (Perna et al., 2005; Boyaci et al., 2007). This allows to trace the difference in perceived size at the level of the local edge activation in V1. Here, we first demonstrate that apparent size 
changes depend on the context of visual information and can be strongly elicited by very short exposure of the stimuli. Then we show that the perceptual size illusion is strongly correlated with the activity in V1. Finally we show that a long-lasting local V1 inhibitory effect can mediate the size illusion.

\section{Materials and Methods}

Participants

Six healthy adults (two females, four males; mean age, 27 years) with normal or corrected-to-normal vision took part in our main experiments (data shown in Fig. $1 B, C$ and subsequent figures). In the additional psychophysical experiments that tested size adaptation aftereffect for different adapter duration, we tested other five subjects, and three of these participated also in the experiment with different adapter-test intervals. Each subject gave informed consent before participation, in accordance with the guidelines of the Human Studies Review Board of the Stella Maris Scientific Institute. Subjects first performed the behavioral experiment outside the scanner and were subsequently scanned for a total of $\sim 3-4 \mathrm{~h}$, over several days ( $3 \mathrm{~h}$ for the main experiment, 30-60 min for the retinotopy and eccentricity localizers).

\section{Visual stimuli}

Visual stimuli were generated in MATLAB (MathWorks), using the Psychophysics Toolbox version 3 extensions. For the behavioral experiments, stimuli were displayed on a Barco Reference Calibrator V CRT monitor (resolution of $1280 \times 1024$ corresponding to $42^{\circ} \times 31.5^{\circ}$ from subject viewing distance of $57 \mathrm{~cm}$ with a refresh rate of $100 \mathrm{~Hz}$ ). For the fMRI experiment, stimuli were presented on LCD goggles (Resonance Technology) with a visual field of $24^{\circ} \times 32^{\circ}$ at a luminance of $\sim 30 \mathrm{~cd} / \mathrm{m}^{2}$. The resolution of the display was $600 \times 800$ pixels, with a refresh rate of $60 \mathrm{~Hz}$. In all experimental conditions, visual stimuli were displayed with an eccentricity of $9^{\circ}$ with adaptors and test stimuli displaced on the left-hand side and the reference (just present in the psychophysical study) on the diametrical opposite position.

The stimuli were Craik-O'Brien-Cornsweet stimuli as shown in Figure 1. The stimuli were constructed by high-pass Gaussian filters (with a $50 \%$ cutoff at spatial frequency of 0.5 cycles $/{ }^{\circ}$ ), with the advantage of eliciting localized activation of the visual cortex, limited to their edges (Perna et al., 2005; Boyaci et al., 2007). To avoid afterimages, the polarity of the stimuli was reversed at $10 \mathrm{~Hz}$. We obtained similar size adaptation aftereffects with a variety of different stimulus configurations (gratings, annuli, homogeneous disks), but we limited for fMRI study to the CraikO'Brien-Cornsweet stimuli given their localized response in V1.

\section{Behavioral experiments}

In the behavioral experiments, the test stimulus was always presented on the left at $9^{\circ}$ eccentricity, whereas the reference stimulus was presented with the same eccentricity on the right. The adapter stimulus was always centered at the same eccentricity as the test. In large adapter blocks, the adapter was $10^{\circ}$, whereas the size of the test was varied by using an adaptive staircase (Quest) on every trial in the range from 0.66 to 1.9 times the size of reference (kept constant at $5^{\circ}$ ). Therefore, the physical size of the test stimulus was always smaller than the adapter. In small adapter block trials, the adapter was $10^{\circ}$, and the size of the test stimulus was varied from 0.66 to 1.9 times the size of reference (kept constant at a size of $15^{\circ}$ ), and thus the test stimulus was always larger than the adapter. Each block consisted of 30 trials, and the order of trials was fully randomized within each block. We collected 30-60 trials for each condition (test $>$ adapter, test $=$ adapter, test $<$ adapter).

The timing of the events and their temporal separation was selected with great care after running several pilot studies. In initial pilot experiments, we used a long adaptation duration ( $40 \mathrm{~s}$ initial adaptation and $7 \mathrm{~s}$ top-up), short separation (300 ms), and short stimulus presentation times $(300 \mathrm{~ms})$, given that adaptation effects are typically strongest in these conditions (Suzuki and Cavanagh, 1998). We then measured the adaptation as a function of temporal separation between the adapter and the test stimulus (Fig. 1D, top). In this condition, the adaptation and top-up duration were $2 \mathrm{~s}$, and the test duration $300 \mathrm{~ms}$. Data for different separations between adapter and test were collected in separate sessions.
For the fMRI experiment, to avoid excessive temporal overlap between responses to adapter and test stimuli, we chose a relatively long temporal separation of $3 \mathrm{~s}$, which still provided a strong effect. We also measured the effect as a function of adaptation duration between 0.5 and $40 \mathrm{~s}$ (Fig. $1 D$, bottom), using a separation and test duration constant of $300 \mathrm{~ms}$ (for adaptation durations larger than $7 \mathrm{~s}$, the subsequent top-up durations were fixed at $7 \mathrm{~s}$, durations smaller than $7 \mathrm{~s}$, and the same initial and top-up durations were used). Based on the finding of these two psychophysical experiments, we chose a relatively short adaptation duration (3 s) for our fMRI stimuli, which allowed us to collect more trials at a shorter time. Using stimuli with timings in which the effect of adaptation is maximum, we would likely have observed a stronger effect of adaptation of that shown in Figures 2 and 3.

Inside the scanner, subjects passively viewed the stimuli without performing a task to avoid an attentive contamination to the adaptation effect and to present a reference stimulus in the contralateral visual field that could elicit an ipsilateral modulation of the response. Visual stimuli (adapter and test) were always presented on the left-hand side to avoid any spatial uncertainty about the location of the stimulus. Subjects were instructed to maintain fixation throughout the experiment and were monitored online with the infrared camera of the goggles. Given that all subjects were trained to keep fixation during the psychophysical testing, we never observed a break of fixation during the stimulus presentation in the scanner. Each trial comprised an adaptation phase (3s) a blank interval ( $3 \mathrm{~s})$, and test interval ( $3 \mathrm{~s})$. Intertrial intervals were varied between 4,5 , or $6 \mathrm{~s}$. In the scanner, we acquired $\sim 45$ trials per condition across several functional runs (see below).

\section{fMRI procedure}

Data acquisition. MRI data was collected on a $1.5 \mathrm{~T}$ scanner (Signa HDxt 1.5 T; GE Healthcare) outfitted with an eight-channel phase array coil. Whole brain functional data were acquired with a single-shot gradientecho, echo planar sequence. Acquisition parameters were as follows: 42 ms echo time (TE); $3000 \mathrm{~ms}$ repetition time (TR); $90^{\circ}$ flip angle; 32 axial slices; $4 \mathrm{~mm}$ slice thickness; $96 \times 96$ matrix; $240 \times 240 \mathrm{~mm}^{2}$ field of view; voxel size of $3 \times 3 \times 3 \mathrm{~mm}$. For the main experiment, we acquired 8-12 functional runs, each consisting of 124 functional volumes (the first four volumes were discarded to allow stabilization of the BOLD signal). Fullbrain anatomical T1-weighted images were acquired for each session of functional data using a three-dimensional fast spoiled gradient recalled echo sequence (TR, $12.3 \mathrm{~ms}$; TE, $2.4 \mathrm{~ms}$; flip angle, $10^{\circ}$; matrix, $256 \times$ $256 ; 1 \times 1 \times 1 \mathrm{~mm}^{3}$ voxel dimensions; 128 slices $)$.

Data analyses. All the data analyses used Brain Voyager QX (version 1.9; Brain Innovation) and MATLAB. After standard preprocessing steps (temporal interpolation and motion correction, as described previously; Crespi et al., 2011), the functional data of each session was realigned and coregistered with the anatomical scans of the same session. Subsequently, for each subject, all sessions were realigned with the high-resolution anatomical scans and were transformed into a standard Talairach coordinate system. To generate flat representations of the cortical surface, the cortex was segmented, inflated, and flattened by geometric projection.

Retinotopic mapping and localizer. We performed separate scans in which we applied classical retinotopic mapping techniques (Tootell et al., 1998) to demarcate the boundaries between areas and to isolate area V1 (white dashed lines in Figure $2 B$ represent the border between area V1 and V2). These maps were acquired by presenting alternating (12 times) horizontal and vertical meridian stimuli (moving dots, $0.33^{\circ}$ diameter, expanding or contracting every $2.0 \mathrm{~s}$, limited lifetime of $300 \mathrm{~ms}$, presented in two opposing sectors, at $\pm 18^{\circ}$ angle) in a 15 s block design, while subjects were instructed to maintain fixation. One run of polar and meridian mapping was recorded for each subject. For eccentricity mapping, a series of counter-phase-flickering annuli, alternating between different radii (from $1.25^{\circ}$ to $20^{\circ}$ ), were used to define $\mathrm{V} 1$ regions that represented different eccentricities (Fig. $3 B$ ). Our subsequent analysis was based on the activity of the cortical surface identified as area V1/V4 and the ROIs of the entire area.

We identified lateral occipital area 1 (LO1) as an area that preferentially responds to intact versus scrambled (Malach et al., 1995; Kourtzi and Kanwisher, 2000, 2001) Craik-O'Brien-Cornsweet stimuli. To lo- 

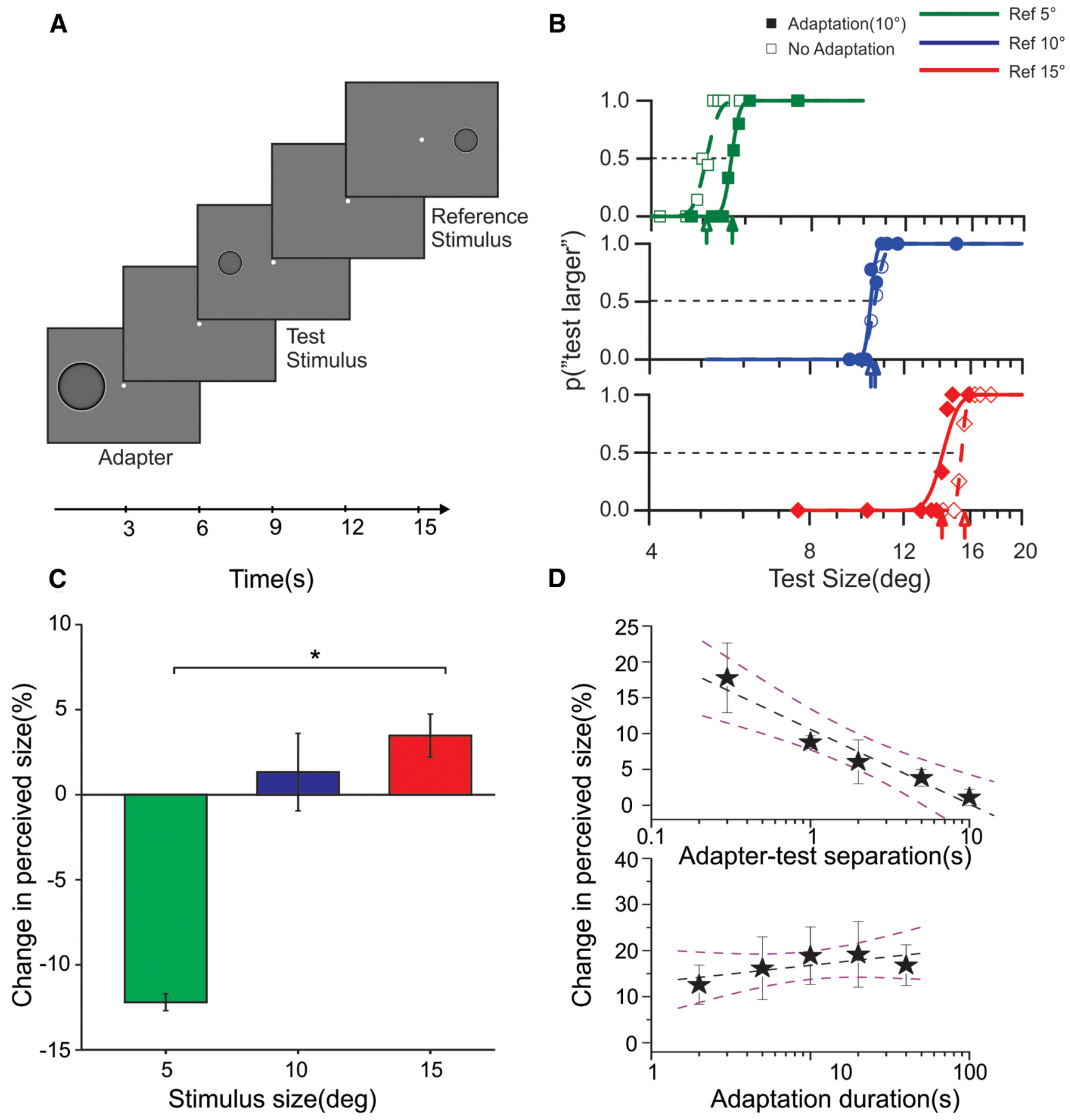

Figure 1. Behavioral experiments. $\boldsymbol{A}$, Subjects discriminated the size of two sequentially presented stimuli (test and reference) displaced horizontally to opposite positions (eccentricity $\pm 9^{\circ}$ ) relative to screen center. The reference stimulus was $5^{\circ}, 10^{\circ}$, or $15^{\circ}$, and the size of the test stimulus varied around that of the reference. The stimuli were bandpass images of filled circles, which induced the Craik- $0^{\prime} B$ rien-Cornsweet illusion (apparent spreading of brightness over the region). In adaptation blocks, the adapter stimulus $\left(10^{\circ}\right)$ was presented before the test centered at the same spatial location. Adapter, test, and the reference stimuli (all at $100 \%$ contrast) and the blanks between them were presented for $3 \mathrm{~s} . \boldsymbol{B}$, Psychometric discrimination functions for $5^{\circ}, 10^{\circ}$, and $15^{\circ}$ of a typical subject: when the size of the reference stimulus was $5^{\circ}$ (top, green), adaptation to a $10^{\circ}$ stimulus resulted in a rightward shift of the psychometric curve, indicating that adaptation decreases the perceived size. When the reference was $10^{\circ}$ (middle, blue), no shift in psychometric curve was observed. With larger stimuli $\left(15^{\circ}\right.$, bottom), adaptation led to a leftward shift, indicating an increase in the perceived size. C, Percentage change in perceived size across all subjects: negative values indicate that stimuli appear smaller after adaptation, and positive values indicate that they appear larger. Stimuli of $5^{\circ}$ and $15^{\circ}$ exhibited a significant change in perceived size with adaptation $\left({ }^{*} p<0.05\right.$, paired $t$ test). D, Decay of the adaptation effect over time (test size, $5^{\circ}$; adapter size, $10^{\circ}$ ). Adaptation strength decreases monotonically with increased temporal separation between adapter and test (top; regression slope $=-10.44 \pm 1.4 \%$ per log-second). Changes in adaptation duration, over a range of $2-40 \mathrm{~s}$, do not significantly affect size illusion (bottom, slope of regression $=3.73 \pm 2.1 \%$ per log-second). The stars depict the average percentage of size change across subjects, the dashed black line is the best linear fit to the data, and the dashed purple lines are the $95 \%$ confidence interval of the fit. Error bars are standard error of the mean (SEM).

calize LO1, we used a blocked presentation design with 24 stimulus epochs (duration 3, TR of 9 s) interleaved with fixation periods (duration 2, TR of 6 s). Stimuli consisted of intact or scrambled versions of CraikO'Brien-Cornsweet stimuli presented in three different sizes $\left(5^{\circ}, 10^{\circ}\right.$, and $15^{\circ}$ ) and in fully randomized order. Stimuli were presented in the left visual field, $9^{\circ}$ eccentricity as had been used in our main experiments. The scrambled images were created by dividing the intact images in a $20 \times 20$ square grid (corresponding to $5^{\circ} \times 5^{\circ}, 10^{\circ} \times 10^{\circ}$, and $15^{\circ} \times 15^{\circ}$ ) 
A
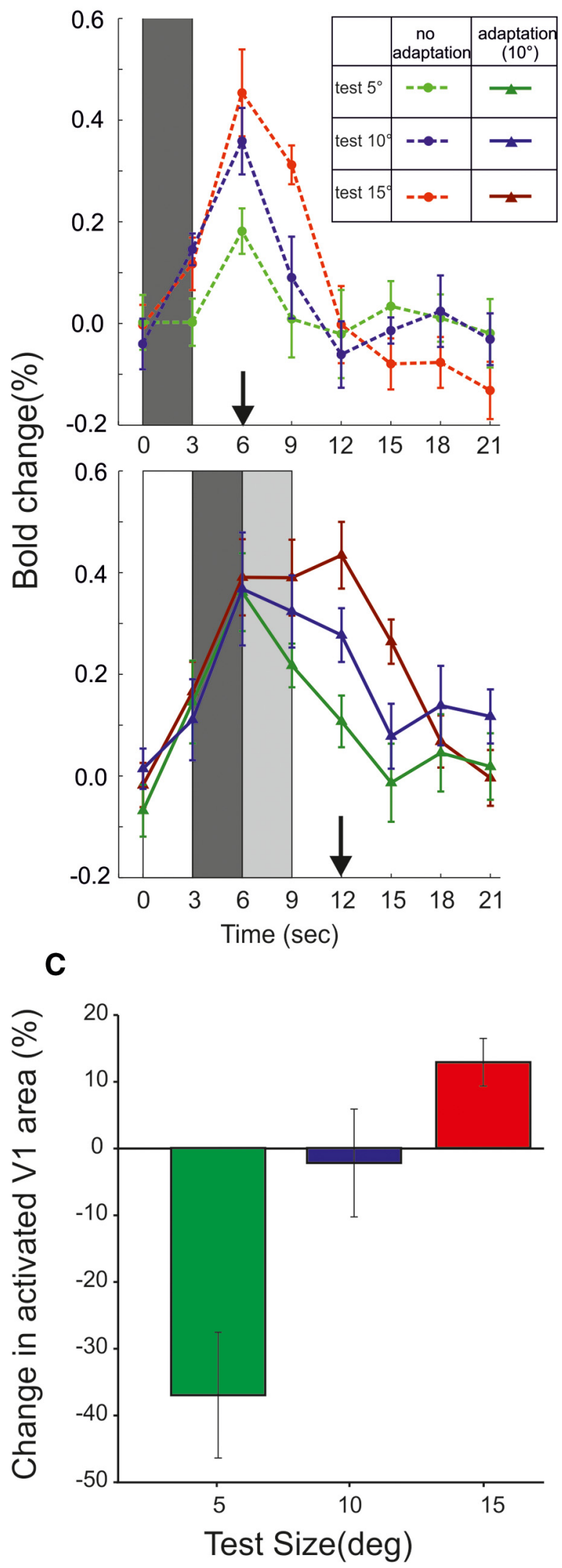

B
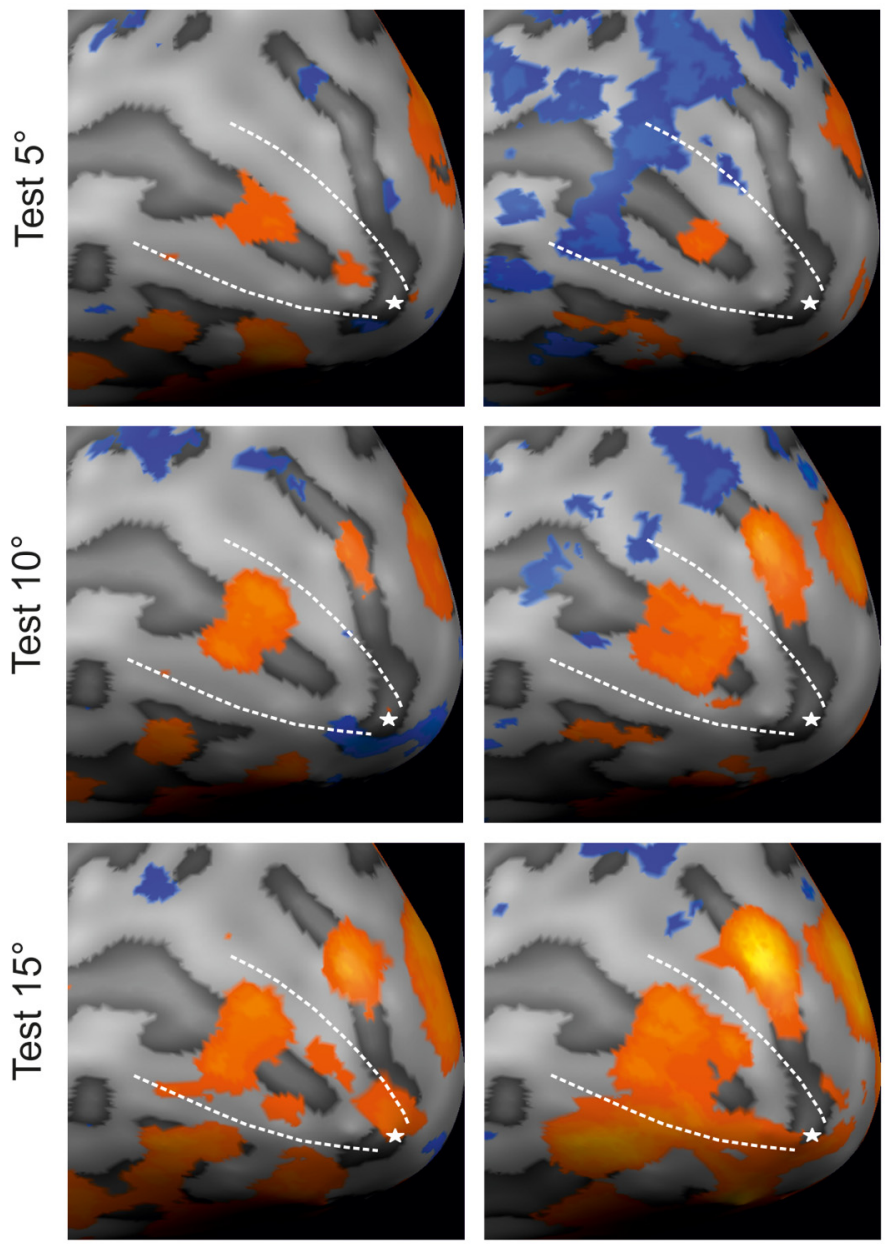

D

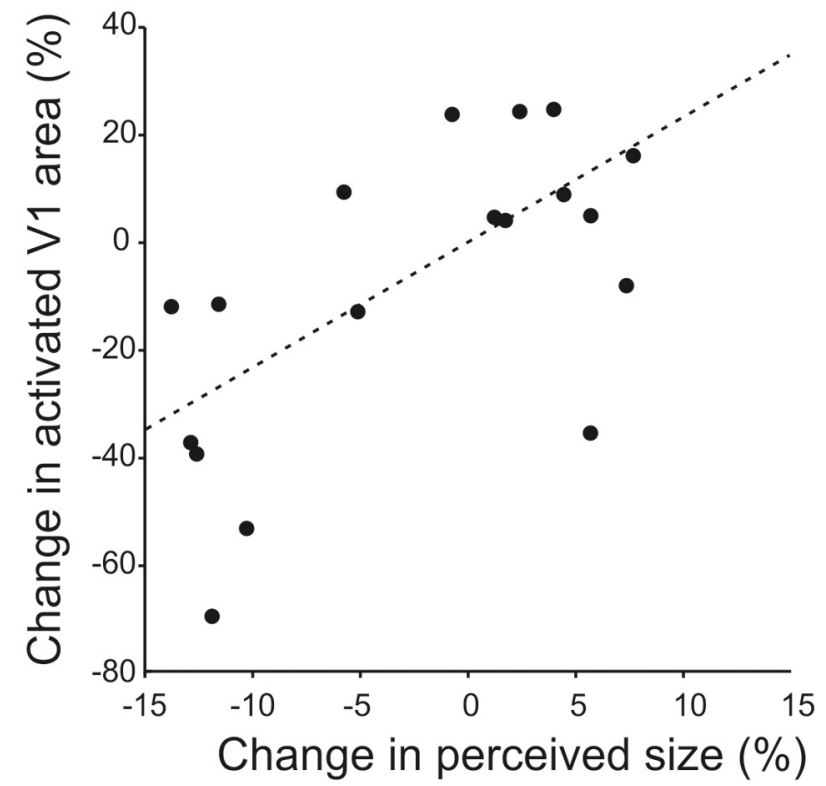

Figure 2. Representation of size illusion in V1 cortex. A, Time course of the event-related V1 activity computed from deconvolved BOLD responses and averaged across all voxels. It should be noted that time courses are from all V1 vertices, defined on anatomical criteria; therefore, higher responses imply a larger spatial extent of activation. The shaded bars indicate the duration of the different events: dark gray, test stimulus; light gray, blank; white, adapter. The responses are aligned to the first event of each trial, i.e., the onset of the test stimulus in no-adaptation blocks and the onset of the adapter in adaptation blocks. The vertical arrows on the abscissa mark the time of maximum responses. B, BOLD activity map of V1 cortex in the adapted and (Figure legend continues.) 
A

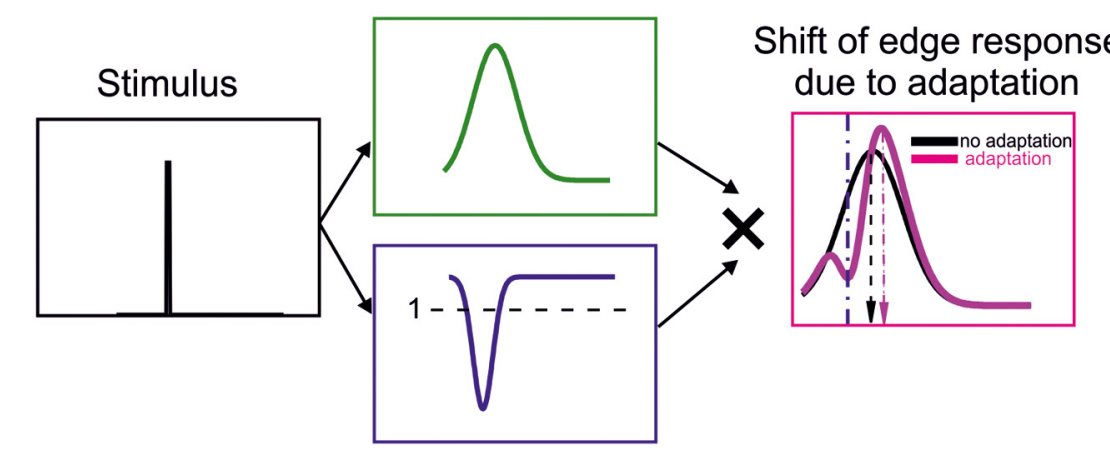

B

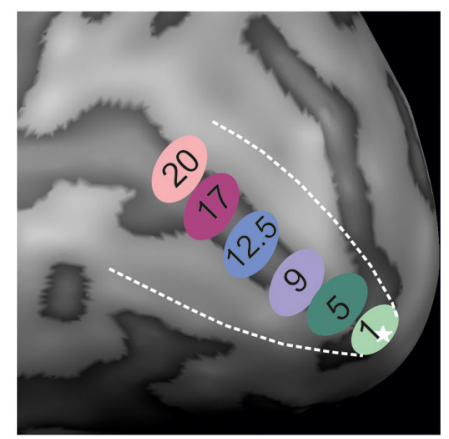

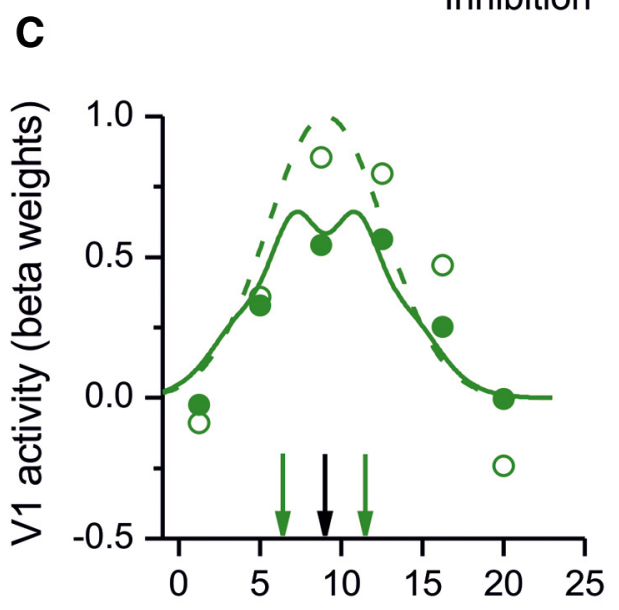
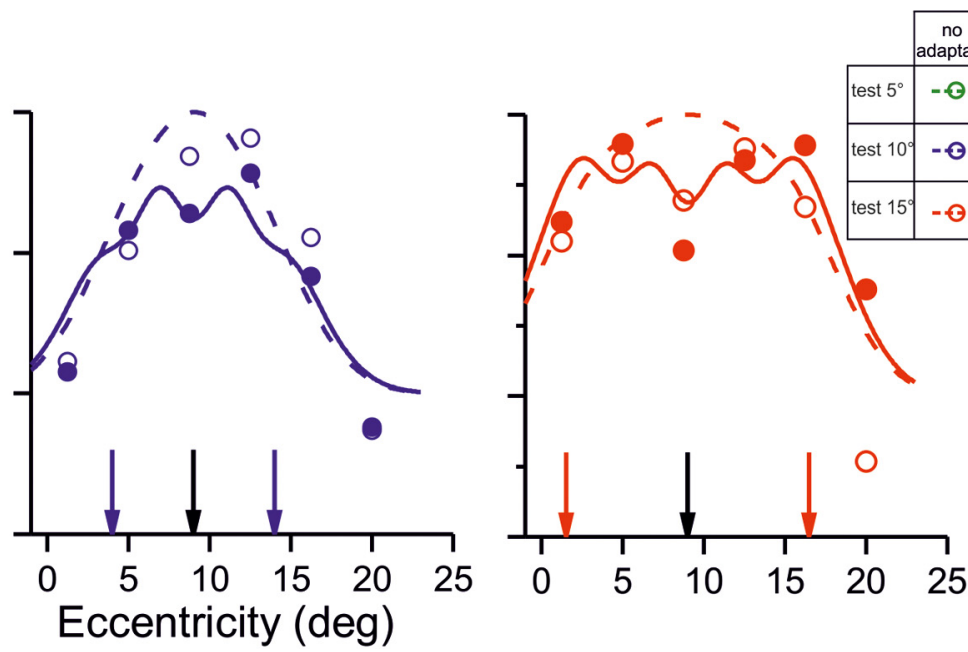

Figure 3. Adaptation effect and its simulation by gain normalization as a function of eccentricity. $A, A$ computational model of the size adaptation effect: after the presentation of the adapter stimulus, cortical areas representing the edges of the adapter undergo suppression, which can be simulated by a response gain modulation as function of cortical space (blue curve). During presentation of the subsequent test stimulus, activation that would normally be elicited by the stimulus (green curve) will be modulated by the gain modulation set by the previous adapter. The multiplication of the two curves will result in a shift in the representation of the stimulus edges away from the adapter. Such a model would predict that, for large stimuli, the edges are shifted outward, whereas for small stimuli, they will shift inward. $\boldsymbol{B}$, To test the model depicted in $\boldsymbol{A}$, responses of six different ROls at different eccentricities were sampled $\left(1.25^{\circ}\right.$ to $\left.20^{\circ}\right) \boldsymbol{C}$, The responses to the stimuli with and without adaptation are shown at each eccentricity with open and filled circles. The vertical black arrow represents stimulus center $\left(9^{\circ}\right.$ eccentricity) and colored arrows the location of stimulus edges at each size. The continuous and dashed curve report the predicted activations based on the model shown in $\boldsymbol{A}$ (for computational details, see Materials and Methods). The curves closely match the measured responses (mean $R^{2}=81 \%$ ).

and by scrambling the positions of each of the resulting squares. The grid lines were present in both the intact and scrambled images. We acquired one run of the lateral occipital complex (LOC) localizer for each subject that consisted of four repetitions of each stimulus configuration (i.e., six configurations: three sizes, either intact or scrambled). In our GLM model, we pooled the responses across different stimulus sizes and contrasted the responses to the intact and scrambled stimuli. We selected LO1 as the portion of LOC that is located anterior to dorsal V3 (Larsson and Heeger, 2006).

Event-related analyses. The adaptation trials comprised four eventsadapter, blank, test (all of $3 \mathrm{~s}$ ), and a blank stimulus of variable and random duration (between 4 and $6 \mathrm{~s}$ ) - whereas for the no-adaptation trials, only the test (duration of $3 \mathrm{~s}$ ) and blank stimuli were presented, again of random duration. Event-related time courses shown on Figure $2 A$ were computed by averaging the event-related responses, with the

$\leftarrow$

(Figure legend continued.) non-adapted condition: the activated cortex shrinks or enlarges

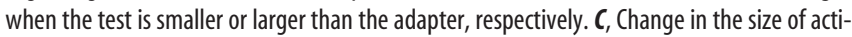
vated V1 cortex (number of vertices suprathreshold) across all subjects, reflecting the decrease, no change, and increase in the size of the activated V1 cortex, when test stimulus was smaller, equal, or larger than the adapter, respectively. D, Correlation between the behavioral and the fMRI effects on each individual subject. Error bars are standard error of the mean (SEM). average of the three time points before the main event serving as the baseline. We performed a linear deconvolution analysis (Burock and Dale, 2000) to separate the responses that were evoked by the test stimulus from responses to the adapter. In this analysis, each trial was modeled as a set of identical time-shifted stimulus impulses [finite impulse response (FIR) functions]. We used eight delta functions, with the first one representing the trial onset (presentation of the test stimulus in no adaptation blocks and presentation of the adapter in adaptation blocks), and the other seven impulse functions representing a single time point of the event-related fMRI time course, each shifted by $3 \mathrm{~s}$ (1 TR). Subsequently, we used a GLM analysis to estimate the overall activity evoked at each time point and at each voxel of area V1. The statistical maps (as shown in Fig. $2 B$ ) represented $t$ values of the FIR regressor that corresponded to the peak response (i.e., third stick function for no adaptation at time $6 \mathrm{~s}$ and fifth stick function for adaptation conditions at time $12 \mathrm{~s}$; these times correspond to the vertical arrows in Fig. 2A). To calculate the area of activated V1 cortex, for each subject, we counted the number of activated vertices (NAVs) on the inflated cortex that were marked at a fixed $t$ threshold. If the $t$ threshold is set at an extremely low value (e.g., $t=-3$ ), all the vertices of the inflated cortex will show an activation that passes this threshold, and NAV will be equal to all V1 vertices. On the other extreme, at highest $t$ values (e.g., $t=5$ ), none of the vertices will be selected $(\mathrm{NAV}=0)$. Between these two extremes, there will be a range of $t$ values in which the NAVs will correctly represent the size of the stimuli 
(i.e., $\mathrm{NAV}_{5^{\circ}}<\mathrm{NAV}_{10^{\circ}}<\mathrm{NAV}_{15^{\circ}}$ ). The final threshold can then be any value within this range. We chose the midpoint of this range [i.e., $0.5 \times$ $\left.\left(\operatorname{Max}_{t}-\operatorname{Min}_{t}\right)\right]$ as the final threshold for each subject [similar results were obtained with different thresholds: from $0.28 \times\left(\operatorname{Max}_{t}-\operatorname{Min}_{t}\right)$ to $0.55 \times\left(\operatorname{Max}_{t}-\operatorname{Min}_{t}\right)$ was used]. The thresholds were derived from the data without adaptation, and the same values were used for the adaptation condition and for all stimuli. The comparisons between NAV in different conditions were done by performing Student's paired $t$ tests. The activities at different eccentricities on V1 (shown on Fig. 3C) were computed from the BOLD amplitude modulation at $6 \mathrm{~s}$ for no adaptation and $12 \mathrm{~s}$ for the adaptation condition (Fig. $2 A$ ) and were averaged across all voxels of an ROI. These activations were used to evaluate our computation model (Fig. 3C).

\section{Modeling the adaptation effect}

We modeled the V1 representation of the test stimuli as elliptical contours whose major axis had the corresponding length of the diameter of the Craik-O'Brien-Cornsweet stimuli used in our experiments. To obtain BOLD cortical simulation, these ellipses $[\operatorname{Input}(x, y)]$ were convolved with a two-dimensional Gaussian:

$$
\text { Activation }(x, y)=\operatorname{Input}(x, y) \times \exp \left(-\frac{x^{2}+y^{2}}{2 \sigma_{\text {act }}^{2}}\right),
$$

where $\sigma_{\text {act }}$ is the width of the activation elicited by an isolated delta function, and $x$ and $y$ are cortical eccentricity along the horizontal and parallel to vertical meridian.

The gain modulation attributable to the preceding adapter was modeled by convolving the adapter ellipse [represented by $\operatorname{Input} A(x, y)]$ with a Gaussian function and is given by the following:

$$
\text { Inhibition }(x, y)=1+\text { gain }-\operatorname{Input} A(x, y) \times \exp \left(-\frac{x^{2}+y^{2}}{2 \sigma_{\text {inhib }}^{2}}\right),
$$

where $\sigma_{\text {inhib }}$ is a space constant.

Adaptation aftereffects were then simulated by a point-by-point multiplication of the activation and inhibition profiles and integrating the two-dimensional spatial response along the horizontal eccentricity to simulate the overall response along iso-eccentricity lines.

The overall response function was modeled by the following:

$$
\text { Response }(x)=\int_{0^{\circ}}^{30^{\circ}} \operatorname{Activation}(x, y) \times \text { Inhibition }(x, y) d y \text {. }
$$

The $\sigma_{\text {act }}, \sigma_{\text {inhib }}$, and gain were the free parameters of our model, and we obtained best fits when they were set to $4.5^{\circ}, 2^{\circ}$, and 0.25 , respectively, for all stimulus conditions.

\section{Results}

Presentation of a spatially high-pass-filtered circle (adaptor stimulus) influenced the perceived size of a similar circle presented successively (the test stimulus; Fig. $1 B, C$ ). When the adapter was larger than the test (adapter, $10^{\circ}$; test, $5^{\circ}$; Fig. $1 B$, top), the psychometric curve, reporting the percentage of perceived larger stimuli in a two-alternative forced-choice discrimination task, was shifted to the right relative to the baseline, indicating a decrease in the perceived size of the test stimulus. Smaller adapters (adapter, $10^{\circ}$; test, $15^{\circ}$; Fig. $1 B$, bottom) had the reverse effect, shifting the psychometric function leftward. When the adapter and the test were the same size (Fig. $1 B$, middle), perceived test size remained veridical, with no shift in the psychometric curves. The effect occurred in all subjects we tested, on average, $12.20 \%$ for larger adapters and 3.5\% for smaller adapters (Fig. 1C).

The amount of adaptation decreased monotonically $(p=$ $0.005, t$ test) with increasing temporal separation between the adapter and the test (Fig. 1D, top), but the effect was still present after $10 \mathrm{~s}$, indicating a prolonged change in neuronal activity, typical of early adaptation phenomena (Maffei et al., 1973). More importantly, the strength of adaptation effect did not vary with the duration of adaptation from 0.3 to $40 \mathrm{~s}$ ( $t$ test for the difference of regression slope from $0, p=0.173$ ), indicating that the temporal dynamics is consistent with the flow of visual information during a single fixation (Fig. $1 D$, bottom). The perceptual effect is strong enough and lasts for sufficient time to be measurable in fMRI experiments, addressing the question whether the size illusion could be traced back to V1 activity.

Inside the fMRI scanner, we measured the BOLD activity elicited by our stimuli in blocks with and without adaptation; subjects passively viewed the eccentric stimuli, without making a size judgment to avoid contamination from the task response or the introduction of a contralateral stimulus reference for the comparison task. In addition, the stimuli were presented intermingled, all attracting the same attentional resource. Figure $2 A$ shows the event-related BOLD responses averaged across all $\mathrm{V} 1$ voxels (defined by individual retinotopy). As expected, the average response of all voxels (including the portion not representing the stimuli) is higher for large compared with small stimuli, reflecting the physical size of the stimulus. The average BOLD modulation of the entire V1 decreases slightly after adaptation for all three stimuli by a similar amount (see arrows at time 6 and $12 \mathrm{~s}$ in Figs. 2A, 4). However, the extent of the activated region over a fixed $t$ threshold (see Materials and Methods) changes differently in the adapted state between small and large size. Figure $2 B$ shows on the inflated cortex of one typical subject the extent of V1 activation in response to different stimulus sizes, with and without adaptation. It can be seen that, when the test stimulus is smaller than the adapter, the area of the activated V1 surface shrinks in size, whereas with test stimuli larger than the adapter, the area of activated V1 surface is enlarged. The activities are consistent with the positions of the border of the stimuli (Fig. 3; Perna et al., 2005). The single focus of activity to the $5^{\circ}$ test after adaptation, positioned between the two foci in the no-adaptation condition, suggests fusion of the two boarder activities in one, consistent with the perception of a smaller stimulus. Analogously, the shift toward more foveal and peripheral location of the foci for the larger-size stimulus after adaptation is consistent with the perception of a larger stimulus. The reduction of the activated area for the smaller stimulus and the increase for the larger stimulus were observed over a wide range of thresholds (from $t=0$ to $t=2$; see Materials and Methods), indicating that the phenomenon is general. To quantify this effect, we counted the NAVs of the inflated cortex of each subject (see Materials and Methods). Across all subjects, we found that adaptation to a larger stimulus significantly decreases the size of the activated V1 cortex by $37 \%$ (paired $t$ test, $p=0.013$ ), adaptation to the same size did not produce any significant change (paired $t$ test, $p=0.13$ ), and adaptation to a smaller stimulus increased the activated surface of V1 by $13 \%$ (paired $t$ test, $p=0.015$ ). Although size adaptation occurred perceptually in all subjects, its magnitude varied considerably, allowing us to look for correlations between the magnitude of the perceptual and the BOLD effects. We observed a significant correlation between change in perceived size of the stimulus and change in the region of activated V1 (Fig. $2 D, r=0.62, p=$ $0.005)$. These results show that the behavioral effects of size adaptation are reflected in the activity of $\mathrm{V} 1$.

To quantify the effect of migration of the foci activity after adaptation (see example in Fig. $2 B$ ), we measured the magnitude of $\mathrm{V} 1$ activation as a function of the eccentricity as illustrated in Figure $3 C$ for the three stimulus sizes, averaged across all subjects. The measured responses to the different stimuli varied with ec- 


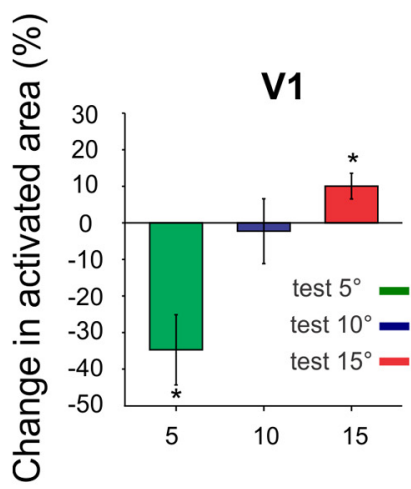

B

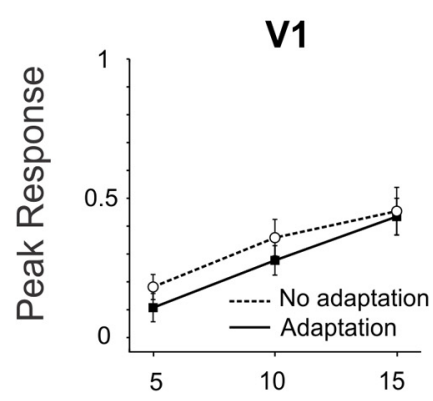

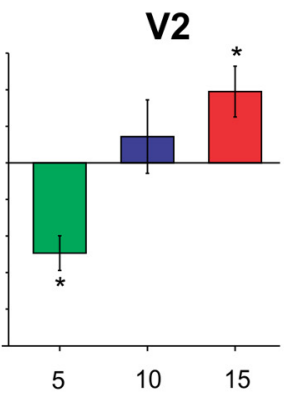

.

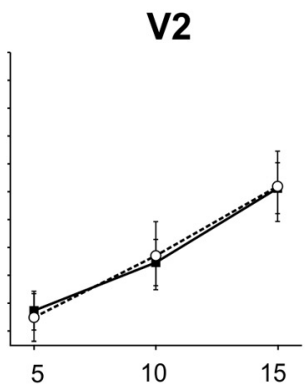

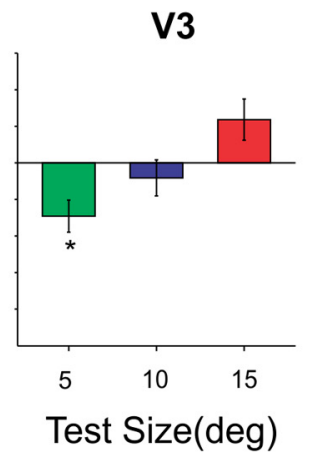
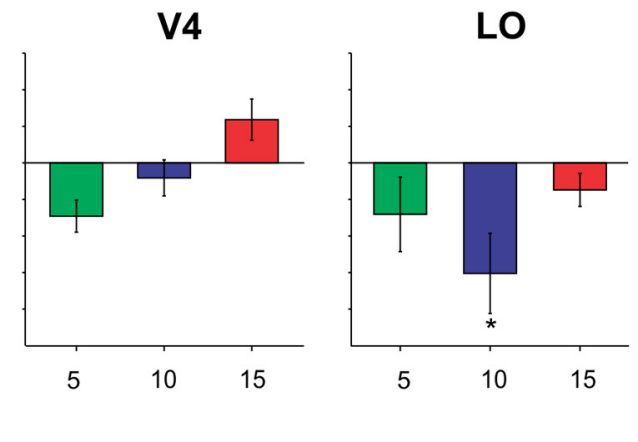

Test Size(deg)

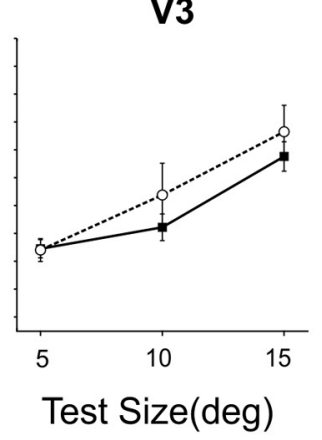

V4

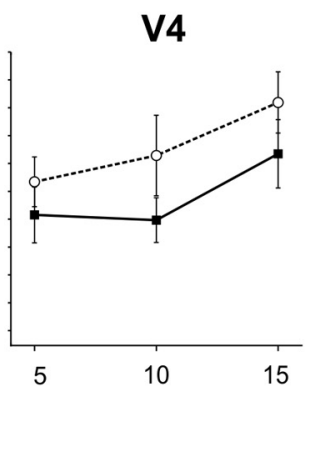

LO

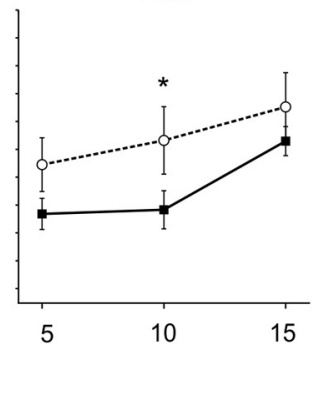

Figure 4. Comparison of adaptation effect in different cortical areas. $\boldsymbol{A}$, Change in the size of activated cortex across all subjects for areas V1, V2, V3, V4, and L0. $\boldsymbol{B}$, Peak responses measured at the time points marked on the abscissa of Figure $2 A$ to the stimuli of different size, with and without adaptation. Test for statistical significance at $p<0.05$, paired $t$ test. Error bars are standard error of the mean (SEM).

centricity (see Materials and Methods), with a significant interaction between stimulus size and the eccentricity at which maximum adaptation effect was observed (repeated-measures ANOVA with difference in adapted and non-adapted responses as dependent variable and stimulus size and eccentricity as independent variables, $\left.F_{(5,2)}=2.04, p=0.048\right)$. This is consistent with the finding that the tuning for the $5^{\circ}$ test (Fig. $3 C$, green data points) becomes narrower after adaptation, whereas that for the $15^{\circ}$ test becomes broader. The adaptation-induced changes of V1 response pattern can be intuitively understood by considering that, after presentation of any stimulus, the cortex becomes less reactive to any subsequent stimulus: a depression trace that persists for a short time, rendering the neurons less likely to be excited by a subsequent stimulus. This phenomenon will generate an interaction between responses elicited by the adapter and the test. We quantitatively modeled the size illusion, hypothesizing that the persistent poststimulus inhibition (Fig. $3 A$, blue curve) modulates response gain. So a border that normally produces responses similar to the green panel of Figure $3 A$ will produce a deformed activity when it follows the presentation of an adapter border at a different position (Fig. $3 A$, magenta curve). The overall effect - the local multiplication of the recent activation (green curve) with the persistent trace (blue curve) - is a centripetal deformation of activity away from the adapter response (for details, see Materials and Methods). If the test stimulus is smaller, this will result in an inward shift for the representation of the stimulus edge, and, if the test stimulus is larger, the representation of stimulus edges will be pushed outward. The model predicts that, for different stimulus sizes, maximum adaptation effect occurs at different locations relative to the adapter edges. We also simulated quantitatively the spatial tuning of responses in both adapted and non-adapted conditions and obtained a good fit to all the data (mean $R^{2}=81 \%$ ), reproducing the observed change in tuning after adaptation.

We have so far shown that activation of area V1 closely matches the behavioral effects of size adaptation and that a response gain model based on local excitation and inhibition within area V1 can simulate our results. We also measured the size adaptation effect in the downstream cortical areas. Given the poorer retinotopic representation of the associative visual cortex with respect to $\mathrm{V} 1$, we focused our analysis on areas in which measurement of the surface of the activated cortex could be reliably performed. We observed a similar and significant increase of activated vertices for large-size test and decrease for smaller-size test than the adapter stimulus in extrastriate areas V2 and V3 (all $p$ values $<0.05$, paired $t$ test). For $\mathrm{V} 4$, the trend was present, but the effect was not significant (Fig. $4 A$ ). Interestingly, the pattern of activation of area LOC, the first area in which objects are categorically represented (Malach et al., 1995; Kourtzi and Kanwisher, 2000), was quite different. In area LO, adaptation resulted in a decrease in the surface of activated cortex for all sizes of the test stimulus with a maximum and significant effect for the stimuli with the same size as the adapter $\left(10^{\circ}\right)$, which is in line with the role of this area in coding object entity (Konen and Kastner, 2008).

The number of significantly activated vertices depends on thresholding and also on amplitude of the BOLD response. Usually, the higher the amplitude of BOLD modulation, the larger the 
NAVs. Interestingly, although the NAVs increased or decreased depending on the relative size of test and adapter (Fig. $4 A$ ), the amplitude of the response modulation, averaged across all the vertices of the anatomically defined visual area, was slightly lower (although not reaching statistically significance) but not different for the different sizes in V1, V2, V3, and V4 (Fig. 4B). In LO1, we observed a significant reduction in the amplitude of the responses when test and adapter stimuli had the same size $\left(10^{\circ}\right)$, corroborating the existence of size-invariant object coding mechanisms in this area (Konen and Kastner, 2008).

\section{Discussion}

We have shown that brief previous presentation of a shape of a certain size changes the perceived size of the succeeding similar shape stimuli. Using shape stimuli that do not contain low spatial frequencies, we were able to show that the activity of V1 correlates with the perceived size of the shape rather than the retinal size. In addition, we were also able to simulate the effect quantitatively by a local inhibitory mechanism that modulates response gain.

Single-unit studies have shown that adaptation changes the sensitivity, tuning, and coding efficiency of neurons at different levels of the cortical hierarchy (Maffei et al., 1973; Dragoi et al., 2000; Georgeson, 2004; Kohn and Movshon, 2004; Gutnisky and Dragoi, 2008). These findings have motivated the proposal that adaptation serves to maximize the efficiency of encoding and the amount of information conveyed by a population of neurons (Barlow, 1990; Clifford et al., 2007). Our results are in line with this theoretical framework, and our proposed model in Figure $3 \mathrm{~A}$ provides a mechanistic explanation based on gain-control mechanisms (Shapley and Enroth-Cugell, 1984) of how this may come about. Gain-control mechanisms are ubiquitous throughout sensory systems and have been identified in the coding of luminance (Enroth-Cugell and Shapley, 1973; Purpura et al., 1990), contrast (Shapley and Victor, 1981; Heeger, 1992; Carandini et al., 1997), and even in more complex perceptual processes, such as face perception (Leopold et al., 2001). Gain-control allows sensors and other mechanisms to adjust sensitivity to the prevailing levels of stimulation, be it light level, contrast, or noise intensity. Typically, inhibitory circuits are responsible for control neuronal gain (Morrone et al., 1982; Heeger, 1992; Carandini and Heeger, 1994). Interestingly, during the awake state, inhibition is predominant and has the role to confine the spread of the evoked response (Haider et al., 2013) in cortical territory and in time. Here we show that gain-control mechanisms, probably through an inhibitory circuit, also underlie more complex perceptual phenomena, such as size adaptation: the tendency to perceive a stimulus larger when it is preceded by a smaller adapter may in fact reflect an automatic adaptation to the prevailing stimulus properties that will enhance the discrimination power for stimuli very similar to the adapter. Through this mechanism, the visual system can increase the amount of information that is conveyed by the neuronal responses (Dragoi et al., 2000). Remarkably, we show that this adaptive coding can be traced back to the spatial BOLD excitation profile of the population responses in area V1. Therefore, adaptation at the level of both single cells and populations of neurons may reflect mechanisms underlying cortical plasticity (Gilbert and Li, 2012) in response to a dynamic environment in which objects may appear in succession and object size scales dramatically with depth. Given the strong evidence that adaptation induces gain changes at the level of single neurons in V1 (Dhruv et al., 2011), it is highly possible that the illusory size effects originate in area V1 and then propagate in a feedforward manner across the cortex. The fact that the size adaptation effect, measured as the NAVs, decreases from V1 to V4 supports this view. However, it is also important to stress that BOLD response in V1 is strongly modulated by feedback signals, and the signal that modulates locally gain response may have a top-down origin, probably from LO, which has selective BOLD tuning for objects (Konen and Kastner, 2008). This view would be consistent with the result that attention is important to elicit in V1 the pictorial size illusion (Murray et al., 2006; Fang et al., 2008). Application of a technique with a higher temporal resolution will be necessary to resolve this issue.

Natural visual scenes are rich in information and continually changing: perception is constantly affected by factors that surround our currently viewed stimulus in space or time (Schwartz et al., 2007). Size adaptation has been considered to be a particular case of figural aftereffects and interpreted as resulting from a shape distortion (Suzuki and Cavanagh, 1998) or motion-related inheritance of spatiotemporal features (Kawabe, 2008). Although these explanations point to the involvement of shape-specific areas, such as the inferotemporal cortex (area IT) or motionrelated areas (area MT+), our data strongly suggest that activity of area $\mathrm{V} 1$, the earliest stage in cortical processing of visual information, may be instrumental in mediating the behavioral effects of size adaptation.

Our results are thus in line with recent findings suggesting that area V1 represents the perceived rather than physical size of a stimulus (Murray et al., 2006; Fang et al., 2008; Song et al., 2011; Sperandio et al., 2012). In the classical design in which contextual size illusions are elicited by perspective or vergence cues, the effect observed in V1 could have been generated in areas that perform cue combination (Cate et al., 2011) and feedback in V1 (Murray et al., 2006; Sperandio et al., 2012). Indeed, optimal cue combination is one of the most powerful models to simulate size illusions (Kersten et al., 2004); the cortical areas that process cue combination might be responsible for computation of the illusory size. In our paradigm, stimuli are presented at the same distance and depth from the observers, so the apparent size could not be attributable to simultaneous spatial contextual effects. It is also unlikely that the illusory perception of size relies on computations performed by higher visual areas and fed back to area V1. Because the effect could be simulated by a strengthening of local inhibition within V1 circuits, it suggests a primary, not auxillary, role of striate cortex. Our results are in agreement with the recent finding that the individual variability of perceived size correlates well with the retinotopic distortion of the V1 map (Schwarzkopf et al., 2011). These findings strongly suggest that V1 activity may be essential in mediating many complex percepts and that the overall role of V1 may be more global, rather than local, in time and space.

Attention is usually an important player in modulating V1 activity (Brefczynski and DeYoe, 1999; Gandhi et al., 1999; Martínez et al., 1999; Somers et al., 1999). To avoid that the attention associated with the judgment of relative size could induce a modification of V1 and generate a response spuriously correlated with the illusion, our subjects did not perform the task during the scans. Given that the adapter and the probe had the same duration, it would have been very difficult for the subject to discriminate between veridical and illusory percepts in the randomly intermingled presentations. In addition, all stimuli would attract the same attentional resources, given that their transient appearance minimized possible attentional interference. The fact that we report a differential effect for small and large stimuli and between two adaptation stages is additional evidence against an explanation based on the attentional state of the subject. Al- 
though attention seems an unlikely explanation for the differential BOLD adaptation change to the small- and large-size stimuli, a possible confound may be associated with the stronger or weaker brightness illusory percepts. It is known that V1 activation correlates with individual subjective brightness (Tsubomi et al., 2012), and this could vary with perceived size (Wachtler and Wehrhahn, 1997) and the apparent contrast of the stimuli (Burr, 1987) that is reduced after adaptation. However, the predicted effects of brightness-induced changes are in the opposite direction. High-contrast edges would induce weaker brightness illusions (Burr, 1987), whereas the no-adapt BOLD response are higher than in the adapted condition. Similarly, larger-size stimuli induce a weaker brightness illusion (Wachtler and Wehrhahn, 1997), although we report an increase in the activated area, not a reduction. Furthermore, the reported variations of the illusory brightness perception are very small, so the presumed contamination in any case would be very small too.

Size illusions have been successfully used to dissociate the systems of vision for action and vision for perception. The dissociation is possible only in particular conditions, in which the perceptual system is more vulnerable to contextual effects (Aglioti et al., 1995). The perceptual system, usually associated with the ventral stream, is more prone to illusory size, and object-size selectivity (Konkle and Oliva, 2012) has been observed along this pathway whereas the vision for action system is usually robust to size illusions (Aglioti et al., 1995). Our result showing that the size aftereffect illusion originates in $\mathrm{V} 1$ predicts that the action system should be prone to the same illusion, as preliminary data from our laboratory seem to suggest.

As already discussed, adaptation is not a phenomenon related exclusively to very long exposure times, because we observed the size illusion for adapters as short as $300 \mathrm{~ms}$. This finding suggests that the effect may be an inherent feature of normal vision that biases the perceived size relative to the size of preceding stimuli. At each fixation (which usually lasts a few hundred of milliseconds), V1 receives different information also about retinal size; for optimal fusion of information across various fixation, temporal context must play a major role in tuning the brain to encode the statistics of the world and recalibrate a subsequent retinal activation on the information of the previous fixation. V1 is probably the most appropriate station for this continuous tuning, given the rich representation of the retinal scene that it contains as we observed here for apparent object size.

\section{References}

Aglioti S, DeSouza JF, Goodale MA (1995) Size-contrast illusions deceive the eye but not the hand. Curr Biol 5:679-685. CrossRef Medline

Andersson F, Joliot M, Perchey G, Petit L (2007) Eye position-dependent activity in the primary visual area as revealed by fMRI. Hum Brain Mapp 28:673-680. CrossRef Medline

Arnold DH, Birt A, Wallis TS (2008) Perceived size and spatial coding. J Neurosci 28:5954-5958. CrossRef Medline

Barlow HB (1990) A theory about the functional role and synaptic mechanims of visual after-effects. In: Vision: coding and efficiency (Blakemore C, ed), pp 363-375. Cambridge, UK: Cambridge UP.

Blakemore C, Sutton P (1969) Size adaptation: a new aftereffect. Science 166:245-247. CrossRef Medline

Blakemore C, Adler K, Pointon M (1990) Vision: coding and Efficiency. Cambridge, UK: Cambridge UP.

Boyaci H, Fang F, Murray SO, Kersten D (2007) Responses to lightness variations in early human visual cortex. Curr Biol 17:989-993. CrossRef Medline

Brefczynski JA, DeYoe EA (1999) A physiological correlate of the "spotlight" of visual attention. Nat Neurosci 2:370-374. CrossRef Medline

Burock MA, Dale AM (2000) Estimation and detection of event-related
fMRI signals with temporally correlated noise: a statistically efficient and unbiased approach. Hum Brain Mapp 11:249-260. CrossRef Medline

Burr DC (1987) Implications of the Craik-O'Brien illusion for brightness perception. Vision Res 27:1903-1913. CrossRef Medline

Carandini M (2000) Visual cortex: fatigue and adaptation. Curr Biol 10: R605-R607. CrossRef Medline

Carandini M, Heeger DJ (1994) Summation and division by neurons in primate visual cortex. Science 264:1333-1336. CrossRef Medline

Carandini M, Heeger DJ, Movshon JA (1997) Linearity and normalisation in simple cells of the macaque primary visual cortex. J Neurosci 17:86218644. Medline

Cate AD, Goodale MA, Köhler S (2011) The role of apparent size in building- and object-specific regions of ventral visual cortex. Brain Res 1388:109-122. CrossRef Medline

Clifford CW, Webster MA, Stanley GB, Stocker AA, Kohn A, Sharpee TO, Schwartz O (2007) Visual adaptation: neural, psychological and computational aspects. Vision Res 47:3125-3131. CrossRef Medline

Crespi S, Biagi L, d'Avossa G, Burr DC, Tosetti M, Morrone MC (2011) Spatiotopic coding of BOLD signal in human visual cortex depends on spatial attention. PLoS One 6:e21661. CrossRef Medline

Dhruv NT, Tailby C, Sokol SH, Lennie P (2011) Multiple adaptable mechanisms early in the primate visual pathway. J Neurosci 31:15016-15025. CrossRef Medline

Dragoi V, Sharma J, Sur M (2000) Adaptation-induced plasticity of orientation tuning in adult visual cortex. Neuron 28:287-298. CrossRef Medline

Enroth-Cugell C, Shapley RM (1973) Adaptation and dynamics of cat retinal ganglion cells. J Physiol 233:271-309. Medline

Fang F, Boyaci H, Kersten D, Murray SO (2008) Attention-dependent representation of a size illusion in human V1. Curr Biol 18:1707-1712. CrossRef Medline

Gandhi SP, Heeger DJ, Boynton GM (1999) Spatial attention affects brain activity in human primary visual cortex. Proc Natl Acad Sci U S A 96: 3314-3319. CrossRef Medline

Georgeson M (2004) Visual aftereffects: cortical neurons change their tune. Curr Biol 14:R751-R753. CrossRef Medline

Gilbert CD, Li W (2012) Adult visual cortical plasticity. Neuron 75:250264. CrossRef Medline

Goodale MA, Milner AD, Jakobson LS, Carey DP (1991) A neurological dissociation between perceiving objects and grasping them. Nature 349: 154-156. CrossRef Medline

Gregory RL (1997) Eye and brain: the psychology of seeing. Princeton: Princeton UP.

Gutnisky DA, Dragoi V (2008) Adaptive coding of visual information in neural populations. Nature 452:220-224. CrossRef Medline

Haider B, Häusser M, Carandini M (2013) Inhibition dominates sensory responses in the awake cortex. Nature 493:97-100. CrossRef Medline

Heeger DJ (1992) Normalization of cell responses in cat striate cortex. Vis Neurosci 9:181-197. CrossRef Medline

Kaufman L, Rock I (1962) The moon illusion. Sci Am 207:120-130. CrossRef Medline

Kawabe T (2008) Spatiotemporal feature attribution for the perception of visual size. J Vis 8(8):7 1-9. CrossRef Medline

Kersten D, Mamassian P, Yuille A (2004) Object perception as Bayesian inference. Annu Rev Psychol 55:271-304. CrossRef Medline

Kohn A (2007) Visual adaptation: physiology, mechanisms, and functional benefits. J Neurophysiol 97:3155-3164. CrossRef Medline

Kohn A, Movshon JA (2004) Adaptation changes the direction tuning of macaque MT neurons. Nat Neurosci 7:764-772. CrossRef Medline

Konen CS, Kastner S (2008) Two hierarchically organized neural systems for object information in human visual cortex. Nat Neurosci 11:224-231. CrossRef Medline

Konkle T, Oliva A (2012) A real-world size organization of object responses in occipitotemporal cortex. Neuron 74:1114-1124. CrossRef Medline

Kourtzi Z, Connor CE (2011) Neural representations for object perception: structure, category, and adaptive coding. Annu Rev Neurosci 34:45-67. CrossRef Medline

Kourtzi Z, Kanwisher N (2000) Cortical regions involved in perceiving object shape. J Neurosci 20:3310-3318. Medline

Kourtzi Z, Kanwisher N (2001) Representation of perceived object shape by the human lateral occipital complex. Science 293:1506-1509. CrossRef Medline 
Larsson J, Heeger DJ (2006) Two retinotopic visual areas in human lateral occipital cortex. J Neurosci 26:13128-13142. CrossRef Medline

Leibowitz H, Brislin R, Perlmutrer L, Hennessy R (1969) Ponzo perspective illusion as a manifestation of space perception. Science 166:1174-1176. CrossRef Medline

Leopold DA, O'Toole AJ, Vetter T, Blanz V (2001) Prototype-referenced shape encoding revealed by high-level aftereffects. Nat Neurosci 4:89-94. CrossRef Medline

Maffei L, Fiorentini A, Bisti S (1973) Neural correlate of perceptual adaptation to gratings. Science 182:1036-1038. CrossRef Medline

Malach R, Reppas JB, Benson RR, Kwong KK, Jiang H, Kennedy WA, Ledden PJ, Brady TJ, Rosen BR, Tootell RB (1995) Object-related activity revealed by functional magnetic resonance imaging in human occipital cortex. Proc Natl Acad Sci U S A 92:8135-8139. CrossRef Medline

Martínez A, Anllo-Vento L, Sereno MI, Frank LR, Buxton RB, Dubowitz DJ, Wong EC, Hinrichs H, Heinze HJ, Hillyard SA (1999) Involvement of striate and extrastriate visual cortical areas in spatial attention. Nat Neurosci 2:364-369. CrossRef Medline

Massaro DW, Anderson NH (1971) Judgmental model of the Ebbinghaus illusion. J Exp Psychol 89:147-151. CrossRef Medline

McCollough C (1965) Color adaptation of edge-detectors in the human visual system. Science 149:1115-1116. CrossRef Medline

Morrone MC, Burr DC, Maffei L (1982) Functional implications of crossorientation inhibition of cortical visual cells. I. Neurophysiological evidence. Proc R Soc Lond B Biol Sci 216:335-354. CrossRef Medline

Murray SO, Boyaci H, Kersten D (2006) The representation of perceived angular size in human primary visual cortex. Nat Neurosci 9:429-434. CrossRef Medline

Perna A, Tosetti M, Montanaro D, Morrone MC (2005) Neuronal mechanisms for illusory brightness perception in humans. Neuron 47:645-651. CrossRef Medline

Purpura K, Tranchina D, Kaplan E, Shapley RM (1990) Light adaptation in the primate retina: analysis of changes in gain and dynamics of monkey retinal ganglion cells. Vis Neurosci 4:75-93. CrossRef Medline

Schwartz O, Hsu A, Dayan P (2007) Space and time in visual context. Nat Rev Neurosci 8:522-535. CrossRef Medline

Schwarzkopf DS, Song C, Rees G (2011) The surface area of human V1 predicts the subjective experience of object size. Nat Neurosci 14:28-30. CrossRef Medline
Shapley R, Enroth-Cugell C (1984) Visual adaptation and retinal gain controls. In: Progress in retinal research, Vol 3 (Osborn NN, Chadler JG, eds). Oxford: Pergamon.

Shapley RM, Victor JD (1981) How the contrast gain control modifies the frequency responses of cat retinal ganglion cells. J Physiol 318:161-179. Medline

Sharpee TO, Sugihara H, Kurgansky AV, Rebrik SP, Stryker MP, Miller KD (2006) Adaptive filtering enhances information transmission in visual cortex. Nature 439:936-942. CrossRef Medline

Somers DC, Dale AM, Seiffert AE, Tootell RB (1999) Functional MRI reveals spatially specific attentional modulation in human primary visual cortex. Proc Natl Acad Sci U S A 96:1663-1668. CrossRef Medline

Song C, Schwarzkopf DS, Rees G (2011) Interocular induction of illusory size perception. BMC Neurosci 12:27. CrossRef Medline

Sperandio I, Chouinard PA, Goodale MA (2012) Retinotopic activity in V1 reflects the perceived and not the retinal size of an afterimage. Nat Neurosci 15:540-542. CrossRef Medline

Suzuki S, Cavanagh P (1998) A shape-contrast effect for briefly presented stimuli. J Exp Psychol Hum Percept Perform 24:1315-1341. CrossRef Medline

Thompson P, Burr D (2009) Visual aftereffects. Curr Biol 19:R11-R14. CrossRef Medline

Tootell RB, Hadjikhani NK, Vanduffel W, Liu AK, Mendola JD, Sereno MI, Dale AM (1998) Functional analysis of primary visual cortex (V1) in humans. Proc Natl Acad Sci U S A 95:811-817. CrossRef Medline

Trotter Y, Celebrini S (1999) Gaze direction controls response gain in primary visual-cortex neurons. Nature 398:239-242. CrossRef Medline

Tsubomi H, Ikeda T, Osaka N (2012) Primary visual cortex scales individual's perceived brightness with power function: inner psychophysics with fMRI. J Exp Psychol Hum Percept Perform 38:1341-1347. CrossRef Medline

Ungerleider LG, Bell AH (2011) Uncovering the visual "alphabet": advances in our understanding of object perception. Vision Res 51:782-799. CrossRef Medline

Wachtler T, Wehrhahn C (1997) The Craik-O'Brien-Cornsweet illusion in colour: quantitative characterisation and comparison with luminance. Perception 26:1423-1430. CrossRef Medline 